\title{
Determinación de capacidad de reposición natural de material pétreo en el tramo del rio Upano denominado la barranca para un manejo de explotación
}

Determination of capacity of natural reposition of stone material in the section of the Upano river denominated the barranca for the design of the operation management

Marco Antonio Mejia. ${ }^{1} \&$ Pedro Quintuña ${ }^{2}$

Recibido: 27-04-2019 / Revisado: 28-05-2019 /Aceptado: 28-06-2019/ Publicado: 15-07-2019

Summary DOI: $\underline{\text { https://doi.org/10.33262/cienciadigital.v3i3.1.711 }}$

The present investigation consisted of establishing the capacity of natural replacement of stone material of the Upano river in the section called the Barranca, which covers a length of $6 \mathrm{~km}$, began with the bathymetric survey, construction of control strips; monthly monitoring for one year at checkpoints; Granulometric physical study of the deposited material, to then establish a model of exploitation of the stone materials, in such a way does not significantly alter a possible environmental use.

Keywords: Bathymetry, Material replacement; stone material

\section{Resumen}

La presente investigación consistió establecer la capacidad de reposición natural de material pétreo del rio Upano en el tramo denominado la Barranca, que abarca una longitud de $6 \mathrm{Km}$, se inició con el levantamiento batimétrico, determinación de franjas de control; monitoreo mensual durante un año a los puntos de control; estudio físico granulométrico del material depositado, para luego establecer un modelo de explotación de los materiales pétreo, de tal manera no altere ambientalmente de manera significativa un posible aprovechamiento.

Palabras claves: Batimetría, Reposición de material ;material pétreo

\section{Introducción}

La importancia económica de los áridos está íntimamente relacionada con el desarrollo socioeconómico de cada país y, consecuentemente, con la calidad de vida alcanzada en

\footnotetext{
${ }^{1}$ Escuela Superior Politécnica de Chimborazo, Ecuador. mejiamarck@yahoo.com

${ }^{2}$ Escuela Superior Politécnica de Chimborazo, Ecuador quintunapeter@ hotmail.com
} 


\section{Cliencia

la sociedad. El río Upano ubicado en la ciudad de Macas por ser uno de los ríos que proporciona una gran parte y por no decir su totalidad de la materia prima (áridos y pétreos) para la construcción, comercialización y elaboración de hormigones y productos artificiales resistentes etc. Debería contar con un estudio técnico que proporcione la información adecuada como es el tiempo de reposición de los materiales, un sistema de explotación acorde a las características in-situ del terreno que permita una explotación ordenada y adecuada de los materiales mineros y que reduzca los impactos que se originan por dicha actividad.

La falta de información y una metodología técnica para la explotación de los materiales está causando que el cauce natural del río Upano se desborde al margen derecho afectando las áreas mineras y asentamientos cercanos

Por lo cual se procede a hacer un levantamiento de información en un tramo de $6 \mathrm{~km}$ a lo largo del río Upano en el sector la Barranca, mediante análisis batimétricos y toma de muestras para ensayos de abrasividad y de granulometría.

\section{Marco teórico referencial.}

\section{Batimetría}

"Es el levantamiento del relieve de Superficies Subacuáticas, ya sea del fondo del mar, como cursos de aguas, lagos, embalses. Se tomarán medidas en X, Y, Z que nos permitan describir el fondo de los mismo, así como sus anomalías presentes a lo largo de su curso de fluido es decir su planimetría y altimetría”. (Pérez \& Merino, 2015).

"La batimetría es la técnica asociada a la obtención de valores de la profundidad de los cuerpos de agua, la cual puede ser de tipo marina, lacustre o fluvial". (Smith \& Sandwell, 1997).

"La información batimétrica posee cuantiosas aplicaciones prácticas como ser la definición de áreas de crecimiento y hábitat de los elementos bióticos, definición de las áreas de distribución de los crustáceos, operaciones de dragado, estudios científicos, seguridad de la navegación marítima, análisis de la diversidad minero metalúrgica, la delimitación de los posibles peligros relacionados con el empleo de instrumentos de pesca y el trazado de cables y tuberías subacuáticas, entre otros". (Calderón, 2002).

\section{Métodos topográficos en batimetría.}

Entre los principales y más usados se destacan:

a) Métodos de posicionamiento planímetro

b) Métodos de posicionamiento altimétrico

\section{a. Métodos de posicionamiento planímetro.}

"El levantamiento es realizado desde la tierra, se realiza como cualquier levantamiento topográfico, pero para determinar la posición planimétrica de un punto, cuya profundidad 


\section{Ciencia

queremos medir es necesario recurrir a procedimientos especiales que han ido evolucionando a medida que la tecnología avanza". (Calderón, 2002).

- Métodos directos.

- Métodos ópticos

- Radicación

- Metodología de GPS

\section{b. Método de posicionamiento altimétrico.}

"Consiste en determinar de la cota de los puntos midiendo la distancia vertical existente entre la superficie del agua y el punto en el fondo, refiriéndose a las coordenadas (X, Y) del punto en el que está situado en el momento de la determinación de la profundidad". (Calderón, 2002).

Por ser uno de los métodos sencillo de realizar, es el más usado para la determinación de la profundidad mínima y máxima en los lechos de los ríos, su desventaja es que hay que tomar varias medidas para sacar un promedio real de la profundidad a medir. Para este caso se puede utilizar una estación total y GPS que nos garantizaran la confiablidad de los datos.

\section{Estación total.}

Es un aparato eléctrico óptimo utilizado para realizar levantamientos topográficos, su funcionamiento se apoya en la tecnología electrónica. Es decir, tiene incorporado un distanció metro eléctrico, entre sus características principales tiene incorporado una pantalla alfanumérica, leds de avisos, un distancio metro trackeador (seguidor de trayectoria) que nos permite ubicar de forma más sencilla la baliza y determinar los niveles de forma electrónica. Este instrumento realiza la medición de los ángulos a partir de marcas realizadas en el los discos o balizas, de igual manera nos permiten obtener coordenadas de puntos respecto a un sistema arbitrario, o sistemas definidos. Para este

proyecto de investigación se utilizó la estación computarizada de marca TRIEMBLE S3 con su respectiva baliza o nivel.

\section{Ensayo de abrasividad o desgaste de la roca.}

"Sirve para conocer el coeficiente de desgaste que tiene los materiales o agregados gruesos de río con la máquina de los ángeles. Se lo realiza generalmente mediante la trituración de los materiales pétreos empleados en las mezclas asfálticas. Este ensayo se lo realiza granulométricamente preparando la muestra que será sometida a abrasión en la máquina de los ángeles y se expresará la perdida de material o desgaste como el porcentaje de pérdida de masa de la muestra en relación a su masa inicial". (Corporación Universitaria de Dios, 2015).

Mediante la siguiente ecuación determinamos el porcentaje de desgaste que ha sufrido la muestra al ser ensayada. 


\section{Silencia Digital}

$\mathrm{P} \%=M i-M f M i * 100$

La representación de los datos se las puede realizar utilizando distintos modelos de tablas, elaborado de acuerdo a la necesidades y utilidad del contratista. Un modelo común se visualiza en la tabla siguiente.

Tabla $\mathrm{N}^{\circ} 1$ Modelo de tabla para ensayos de desgaste

\begin{tabular}{|c|c|c|c|c|c|c|c|}
\hline \multirow{3}{*}{$\begin{array}{c}\text { Tamaño de } \\
\text { particulas (mm) }\end{array}$} & 1 & 2 & 3 & 4 & 5 & 6 & 7 \\
\hline & $(80-40)$ & $(50-25)$ & $(40-20)$ & $(40-10)$ & $(20-10)$ & $(10-5)$ & $(5-2,5)$ \\
\hline & \multicolumn{7}{|c|}{ Tamaño de las fracciones (g) } \\
\hline $80-63$ & $2500=50$ & & & & & & \\
\hline $63-50$ & $2500=50$ & & & & & & \\
\hline $50-40$ & $5000=50$ & $5000 \pm 50$ & & & & & \\
\hline $40-25$ & & $5000=25$ & $5000 \pm 25$ & $1250=25$ & & & \\
\hline $25-20$ & & & $5000 \pm 25$ & $1250 \pm 25$ & & & \\
\hline $20-12,5$ & & & & $1250=10$ & $1250 \pm 10$ & & \\
\hline $12,5-10$ & & & & $1250 \pm 10$ & $1250 \pm 10$ & & \\
\hline $10-6,3$ & & & & & & $1250=10$ & \\
\hline $6,3-5$ & & & & & & $1250 \pm 10$ & \\
\hline $5-2,5$ & & & & & & & $5000=10$ \\
\hline $\begin{array}{l}\text { Masa inicial de } \\
\text { muestra (Mi) }\end{array}$ & $10000=100$ & $10000=75$ & $10000 \pm 50$ & $5000=10$ & $5000=10$ & $5000=10$ & $5000=10$ \\
\hline \multicolumn{8}{|l|}{ Esferas } \\
\hline -Número & & 12 & & 12 & 11 & 8 & 6 \\
\hline -Masa(g) & & $5000=25$ & & $5000=25$ & $4584 \pm 25$ & $3330=25$ & $2500=15$ \\
\hline $\begin{array}{l}\text { Número de } \\
\text { Revoluciones }\end{array}$ & & 1000 & & & & & \\
\hline
\end{tabular}

Tomada de Corporación Universitaria de Dios, 2015

\section{Ensayo granulométrico.}

Las propiedades físicas y químicas de los sólidos varían de acuerdo a la distribución granulométrica de los materiales. Para poder efectuar controles de calidad en los materiales granulados es indispensable conocer la distribución del material de acuerdo al tamaño de partículas que lo conforman.

"Para la realización de este ensayo se utiliza una serie de distintos tamices, de distintos tamaños que van desde 2", 1", 3/4", 4",8",16",32" de esta forma se podrá determinar la composición granulométrica del material y su respectiva clasificación de acuerdo a su tamaño". (Universidad Sucre,2007).

Tabla N2 Clasificación del material de acuerdo al tamaño de partícula. 


\begin{tabular}{ccc}
\hline Tipo & Clasificación & Tamaño \\
\hline Bloques & & $>63 \mathrm{~mm}$ \\
\hline \multirow{2}{*}{ Gravas } & Grueso & 63 a $20 \mathrm{~mm}$ \\
\cline { 2 - 3 } & Medio & 20 a $6,3 \mathrm{~mm}$ \\
\cline { 2 - 3 } & Fino & 6.3 a $2 \mathrm{~mm}$ \\
\cline { 2 - 3 } Arena & Grueso & $2 \mathrm{~mm} \mathrm{a} 0.63 \mathrm{~mm}$ \\
\cline { 2 - 3 } & Medio & 0.63 a $0.2 \mathrm{~mm}$ \\
\hline
\end{tabular}

Tomada de Corporación Universitaria de Dios, 2015

\section{Hidrología.}

El río Upano es de tipo anastomosado o río terrazado, es decir que es un río constituido por una serie de canales a lo largo de su cauce, haciendo que en este se formen pequeños bancos o terrazas, provocando que el cauce de río presente variaciones en sus direcciones que caracteriza a este tipo de ríos. Una de las características principales que posee el río Upano son sus bancos y canales trenzados, que conglomeran grandes cantidades de sedimentos detríticos de interés trasportados desde la cordillera de tipo volcánico (áridos y pétreos) dichos bancos son variables ya que dependen de las crecientes del río.

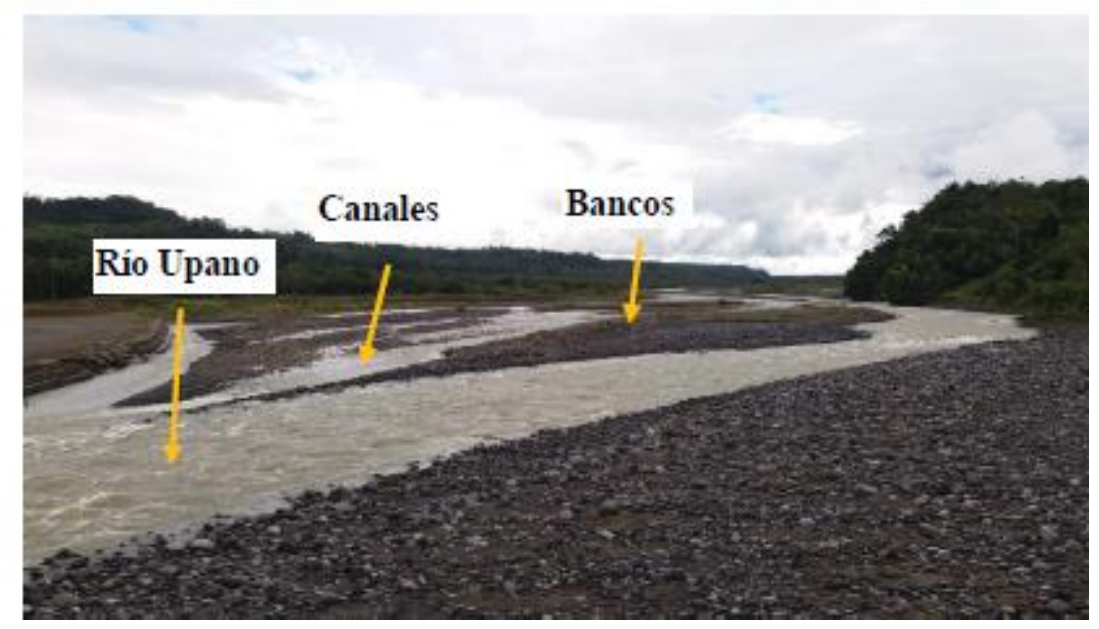

Grafico $N^{\circ} 1$ descripción del Rio Upano

\section{Explotación.}

"Comprende el conjunto de operaciones, trabajos y labores minerales, destinadas a la preparación y desarrollo de la cantera y a la extracción y transporte de los materiales áridos y pétreos". (Herrera \& Pla, 2006). 


\section{Cliencia

Las técnicas de explotación están ligadas directamente a las características del material, maquinaria a utilizar, volumen de extracción y a la normativa vigente de la ley minera con respecto a los volúmenes máximo de extracción permitidos para la minería artesanal.

\section{Materiales de Construcción.}

"Los materiales de construcción, consisten en rocas y derivados de las rocas, sean estas de naturaleza ígnea, sedimentaria o metamórfica tales como: andesitas, basaltos, dacitas, riolitas, granitos, cenizas volcánicas, pómez, materiales calcáreos, arcillas superficiales; arenas de origen fluvial o marino, gravas; depósitos tipo aluviales, coluviales, flujos laharíticos y en general todos los materiales cuyo procesamiento no implique un proceso industrial". (Herrera \& Pla, 2006).

\section{Marco Metodológico \\ Descripción del área.}

Se tomó en cuenta como punto referencial, (punto 0) al puente del río Upano; el área constituida de $1 \mathrm{~km}$ se denomina Zona A; y el área conformada por $5 \mathrm{~km}$ se denomina aguas abajo se denomina Zona B ; se realizó un levantamiento de información para conocer las características actuales del mismo. Donde se pudo comprobar lo siguiente:

En Zona 1 se visualiza una gran cantidad de material acumulado en bancos distribuidos en todo su ancho, lo que forma canales por donde circula el cauce del río. A medida que se avanza 500 metros aguas abajo se puede observar intervención antrópica, la misma que ha ocasionado una variación en la dirección de flujo natural del río, de igual manera la reposición natural del material se lo hace de manera irregular, visualizándose islotes de grandes tamaños en zonas donde el cauce del río no circula. Todas estas irregularidades en el cauce del río producto de una explotación irregular se observan en un tramo total de 100 metros aguas abajo.

Un kilómetro y medio aguas abajo se puede observar que el cauce natural del río ha sufrido una desviación ya que en su totalidad es direccionado hacia el margen derecho, en dirección de avance del mismo, este encausamiento es realizado por maquinaria de los GAD, se visualiza que el principal motivo es el aprovechamiento del material en esa zona puntal, puesto que a unos 500 metros más abajo, el río sigue retomando su curso sin ninguna variación, debido a que no hay áreas presentes que sean usadas para extraer material pétreo.

En el recorrido que se realizó de los 6 kilómetros se pudo comprobar un número cercado a 12 permisos de minería artesanal, destinadas a la explotación de los materiales pétreos, las cuales en su mayoría limitan con las riberas del río, lo que ocasiona que el río altere su cauce en algunos tramos producto de la explotación mal llevada de los mineros artesanales. 


\section{Ciencia

\section{Fisiografía del material}

Predominan aquellos de un diámetro entre los menores a $2 \mathrm{~cm}$ como son las arenas, 2 a $30 \mathrm{~cm}$ y hasta bloques de 40 a $80 \mathrm{~cm}$, conocidos como guijarros que generalmente son usados para formar escolleras a la hora de explotar el mismo.

La capacidad de arrastre de sedimentos detríticos que tiene el río Upano en las crecentadas es alta, ya que se pudo observar la presencia de bloques de grandes dimensiones y redondeados lo que demuestra la gran distancia que han sido arrastrados

\section{Procesado de los datos Batimétricos.}

Para realizar el levantamiento batimétrico se consideró dos zonas específicas de $600 \mathrm{~m}$ aproximadamente la primera y $400 \mathrm{~m}$ aproximadamente la segunda; la primera fue elegida por ser considerada como zona de exclusión minera por encontrarse cerca de una obra civil sensible; la segunda se optó por ser una zona de características favorables para la explotación y por contar con el permiso de los concesionarios.

El fin primario es determinar la profundidad máxima de explotación del río, que permita estimar cual es el volumen de material posible a extraer, así como su volumen de reposición natural del mismo.

Para el levantamiento de los tramos restantes se recurre a una base topográfica, donde se delimita una tercera zona destinada a la realización del muestreo para los ensayos físico mecánicos.

Una vez identificada las zonas para el estudio batimétrico, se hace el levantamiento topográfico y el levantamiento batimétrico, con la estación computarizada de marca TRIEMBLE S3 con su respectiva baliza o nivel; desde un punto de partida inicial en cada zona, se procede a tomar los datos a lo largo del tramo que está circunscrito dentro del mismo. Este levantamiento se realizó en los meses de octubre y diciembre del 2018

Para realizar el levantamiento topográfico se dividió en distintas etapas. La primera etapa Para realizar el levantamiento topográfico se dividió en distintas etapas. La primera etapa fue la ubicación de los BM o punto de partida; en este caso los BM se encuentran ya determinados y ubicados en puntos que nos permitieron tener un mayor grado de observación de la zona total a estudiar. Una vez establecido el punto de partida se procede con el armado de la estación total.

Se tomó un total de 78 puntos en total del área de estudio seleccionado, que permitió de esta forma obtener un mayor grado de precisión en el levantamiento realizado. Una vez completo el levantamiento de toda el área seleccionada de estudio se procedió a extraer la base de datos en el software especializado, para este caso se utilizó el AutoCAD 2018. Como metodología para determinar la reposición del río Upano en lapso de un año, la topografía antes mencionada se somete a comparación con otro levantamiento topográfico realizado en diciembre del 2017. 


\section{OCiencia

Para este efecto se utilizó las herramientas del autodesk denominado civil 3 D el cual genera un modelo o simulación producto de la comparación entre estos dos levantamientos topográficos. Obteniendo un resultado en volumen de material.

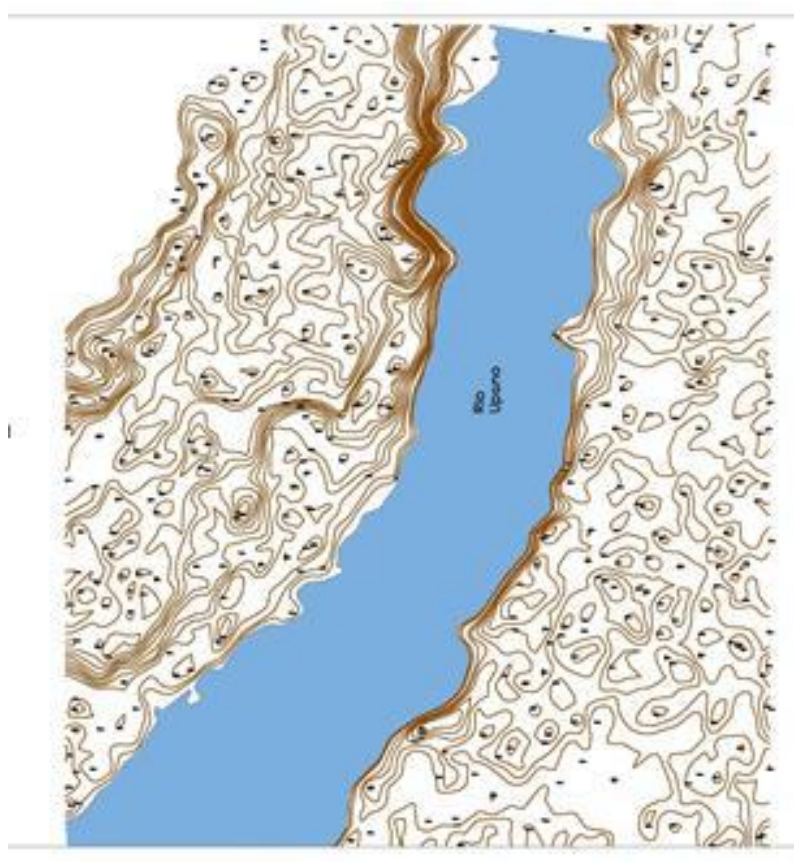

Gráfico $\mathrm{N}^{\circ} 2$ Base topográfica de la zona de estudio

\section{Ensayo de abrasividad.}

Para determinar el grado de desgaste que tienen los sedimentos de río, producto del arrastre del cauce del mismo, se realizó un ensayo de laboratorio utilizando una máquina de los ángeles, para lo cual se siguieron los siguientes pasos:

Se identificó el área donde se procedió a tomar muestras representativas de la zona.

- Se realizó la recolección de muestras en distintos sacos con un peso aproximado de $25 \mathrm{~kg}$.

- Las muestras recolectadas fueron llevadas al laboratorio, donde fueron lavadas y se realizó un roleo de la misma para obtener una muestra homogénea, para depuesto ser sometidas al ensayo.

- Para la ejecución del ensayo se siguieron los siguientes pasos:

- Se hace pasar un total de 1250 gr de muestra por el tamiz $1 \frac{1}{1 / 2}$ pulgada, luego un total de 1250 gr por un tamiz de 3/4, en el tamiz de $1 / 2$ también un total de $1250 \mathrm{gr}$ y por ultimo de $3 / 8$ hasta obtener un total de 5000 gr de muestra.

- Los 5000 gr de muestra se introducen al molino de ángeles, se adiciona un total de 12 bolas y se lo deja moler a una velocidad operacional de 30 a $33 \mathrm{rpm}$ por un tiempo de 15 minutos.

- Extraemos la muestra producto de la molienda y se realiza la comparación entre la masa inicial y la masa final y se obtiene el porcentaje de desgaste del mismo. 


\section{ocances

Para la determinación del porcentaje de desgaste se utilizó un formato establecido por el laboratorio del Consejo Provincial, donde considera: tipo de material, masa inicial, masa final, \% de abrasividad

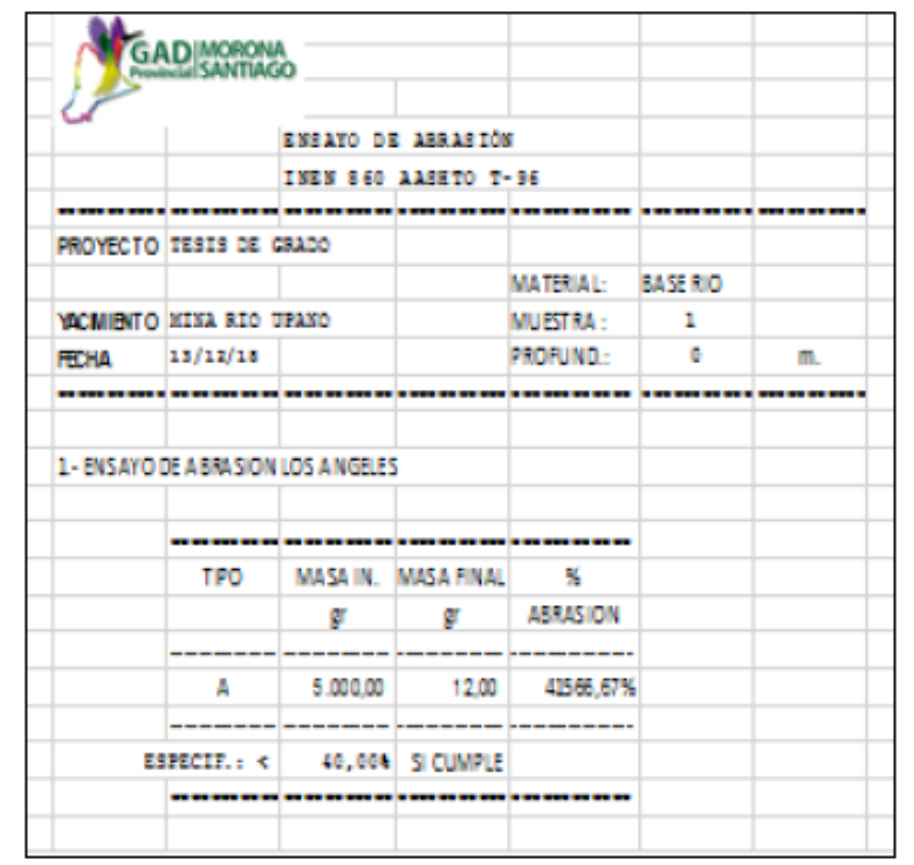

Tabla 3: Modelo de tabla del laboratorio para ensayo de abrasión.

El resultado de este ensayo servirá para saber la calidad y el grado de desgaste que tiene este material y determinar si cumple los parámetros establecidos por la NEVI, donde establece un rango de desgaste no mayor al $40 \%$ de la masa inicial para materiales que serán usados como base y sub-base en la ejecución de obras civiles.

\section{Ensayo granulométrico.}

Para la obtención de las muestras que serán sometidas al ensayo de granulometría se realizó un recorrido de una sección del río, donde se extrajo $1 \mathrm{~m} 3$ de material. Una vez extraído el material, se procede a realizar el pesado y tamizado del mismo, ya que del $100 \%$; el $70 \%$ corresponde a material mayor a $15 \mathrm{~cm}$ de diámetros y el $30 \%$ corresponde a material inferior a estos diámetros, dicho análisis se pudo comprobar haciendo pasar el material por una criba.

Del metro cúbico de la muestra, el peso total de esta es de $1480 \mathrm{~kg}$; el $70 \%$ denominado material grueso equivale en peso a $1030 \mathrm{~kg}$; y de material fino que corresponde al $30 \%$ es de $440 \mathrm{~kg}$.

Al material fino (30\%) se realizó una homogenización mediante roleo para extraer $2.5 \mathrm{~kg}$ de muestra bruta (representativa), para someterla al ensayo granulométrico en los tamices de $63 \mathrm{~mm}, 31.5 \mathrm{~mm}, 16 \mathrm{~mm}, 8 \mathrm{~mm},<4 \mathrm{~mm}$, en los cuales se hizo pasar los $2.5 \mathrm{~kg}$ de muestra por los 4 tamices y se pesó el retenido en cada tamiz. 


\section{Ciencia

De esta manera pudimos realizar una clasificación total del metro cúbico analizado, basada en una clasificación práctica y recomendada por el laboratorio del consejo provincial, en donde clasifican al material en grueso y fino, en el que todo el material mayor a $64 \mathrm{~mm}$ es considerado como gravas, y el material menor a ese diámetro como arenas.

Una vez realizado el pesado de los pasantes de cada uno de los tamices utilizados se construye la curva granulométrica de la muestra analizada, utilizando una tabla modelo diseñada por el laboratorio del consejo provincial de Morona Santiago.

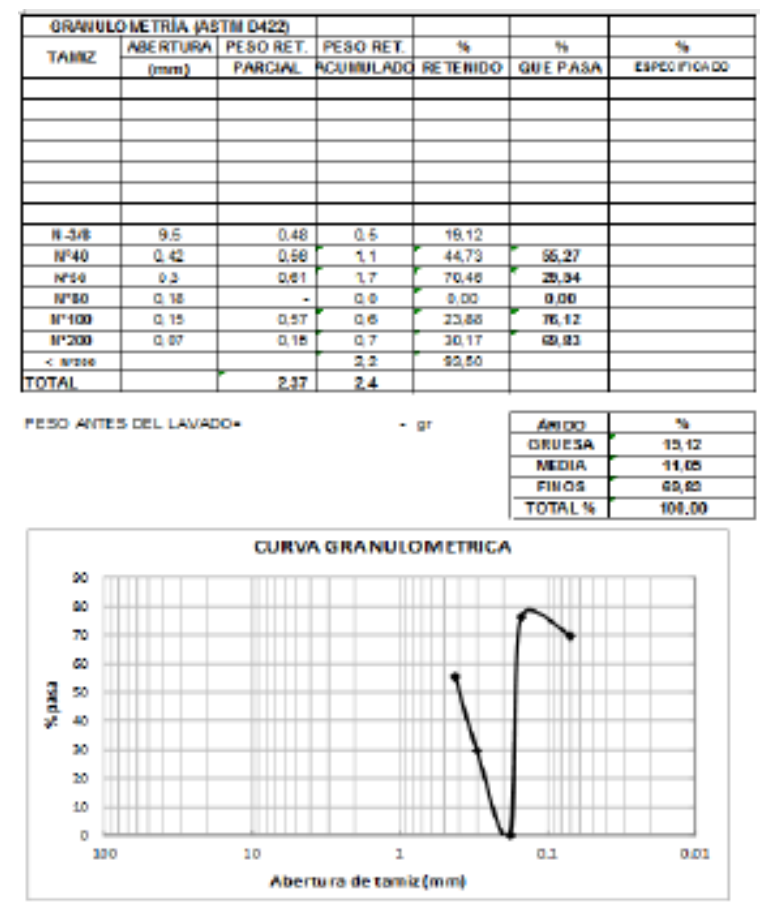

Tabla 4: Modelo de tabla del laboratorio para ensayo de abrasión.

\section{ANÁLISIS E INTERPRETACIÓN DE RESULTADOS.}

Una vez realizado el levantamiento de la zona seleccionada y el recorrido de toda la sección de estudio, se presentan los resultados de acuerdo a los objetivos planteados.

\section{Análisis de los cortes Batimétricos}

Para una mejor comprensión del tramo de estudio se procede a realizar la interpretación de dos cortes longitudinales; zona A (aguas arriba) y la zona B (Aguas abajo), tomando como punto de referencia el puente del río Upano. En los cuales se podrán visualizar las variaciones que presenta la rasante del río dentro de la zona de estudio.

\section{Análisis del corte batimétrico de la zona A (Aguas arriba)}

En el corte batimétrico Grafico 3), se observa una pendiente relativamente suave obteniendo así una profundidad máxima en dicha zona que es de $3.40 \mathrm{~m}$ con variaciones a lo largo de su curso alcanzando profundidades mínimas de $1.5 \mathrm{~m}$ en relación a su espejo 


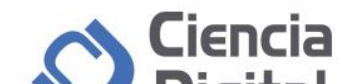 Digital}

de agua, en vista a las características que presenta el río Upano el cual en este sector posee unas características isométricas siendo su elevación de la superficie la misma en ambos lados de la playa.

PERFLL AGUAS ARRBB

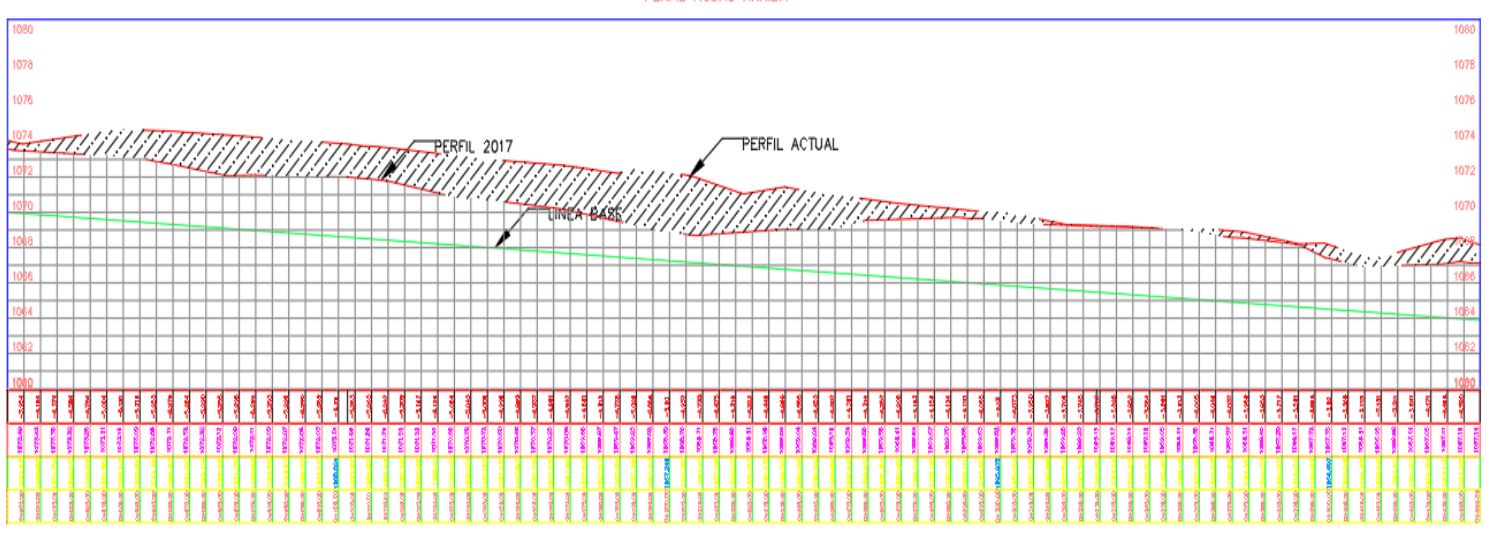

Grafico 3: Corte Batimétrico de la zona A (Aguas arriba) Análisis del corte batimétrico de la zona B (Aguas abajo)

En el corte batimétrico (ilustración 4), se puede observar que esta zona posee características similares a la zona A, ya que el material y las condiciones en la que se encuentra depositado son las mismas por lo que no hay variación significativa en su longitud total.

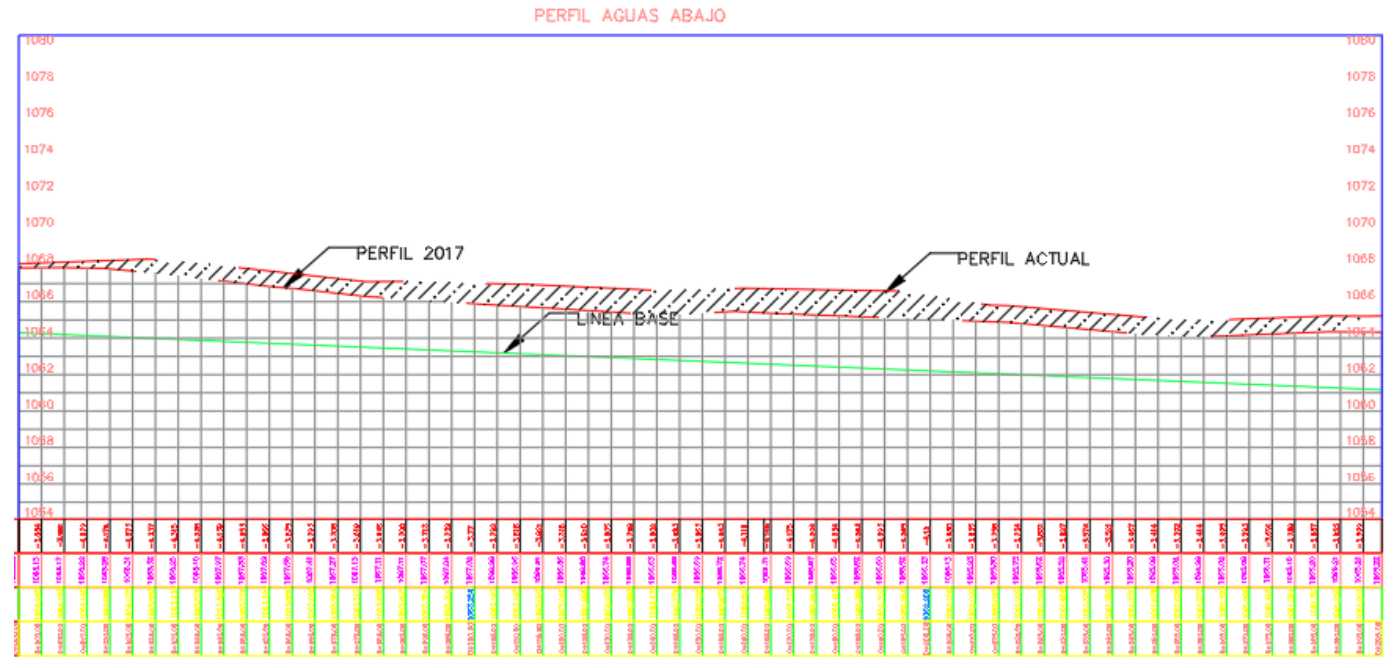

Grafico 4: Corte Batimétrico de la zona B (Aguas abajo) 


\section{Ciencia

\section{Resultados de la batimetría.}

Con los resultados obtenidos del levantamiento del área seleccionada, se pudo comprobar la cantidad de material que se puede extraer de una sección del río, así como la capacidad de reposición que tiene el mismo en las distintas crecentadas en un período de 9 meses. En el primer tramo aguas arriba, en una piscina de explotación realizada de 40 metros de largo por 20 de ancho y con una profundidad de 3.40 metros se estimó un aproximado de la reserva de $68162.32 \mathrm{~m} 3$ de material y en el tramo dos, aguas abajo en una sección de 20 metros de ancho por 20 metros de largo y una profundidad similar, se estimó una cantidad de $39419.81 \mathrm{~m} 3$ de material pétreo.

Para comprobar la capacidad que tiene el río de reponer el material de manera natural, se realizó una piscina donde se extrajo el material hasta una profundidad de 3.40 metros y se trazó una línea base en la rasante, se esperó por un período de tiempo estimado de 9 meses, con controles mensuales y se volvió hacer un levantamiento para comprobar el nivel actual del material y de esta forma calcular la cantidad de material que repuso el río en todo ese período de tiempo.

Con el levantamiento final se puede verificar la capacidad que tiene el río de reponer el material de forma natural en las distintas crecentadas y lo viable que es continuar explotando dicho material de una manera técnica y responsable con el medio ambiente.

Con la línea base trazada la primera vez y con una comparación con la línea actual realizada el 9 de diciembre del 2018 se pudo estimar una reserva de material zona A de $107952.75 \mathrm{~m} 3$ de material y Zona B con volumen de $21029.38 \mathrm{~m} 3$ de material. Comparando los volúmenes de material con los actuales se estima que, en un período de 9 meses desde el primer levantamiento en el 2017, el río repuso un volumen superior al extraído en un total de $60819.81 \mathrm{~m} 3$ de material adicionales a lo inicial. Con la información obtenida con la que se realizó la batimetría en toda esta sección, podemos decir que en el río Upano en las distintas temporadas tanto invierno como de verano repone un aproximado mensual de $14331.34 \mathrm{~m} 3$ de material.

Se trazaron 32 perfiles en total, lo que permitió visualizar el porcentaje de material de reposición, realizando un perfil de cada abscisa y aplicado el método prismoidal para el cálculo de volumen que aplica la fórmula.

\section{$\mathrm{V}: L 3(A 1+\sqrt{ } A 1 A 2+A 2)$}

Para poder comprobar de una manera gráfica la forma en la que se depositó el material en todo el tramo de estudio, se realizó unos perfiles en las 6 abscisas, de esta forma se podrá visualizar el área de corte de cada sección y el volumen de corte aproximado que se obtendrá en dicho tramo, para que en caso de una posterior extracción saber en qué secciones se encuentra más material.

En la sección aguas abajo se realiza una comparación, los levantamientos del 2017 frente a los levantamientos del 2018. 


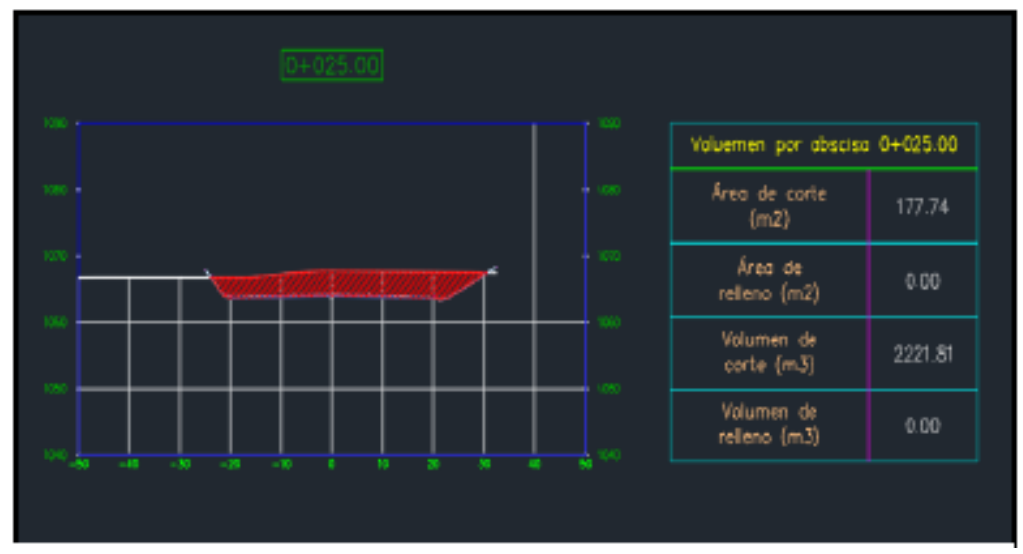

Grafico 5: Perfil aguas abajo 2018, abscisa 0+075.00. total 32 perfiles

\begin{tabular}{|c|c|c|c|}
\hline \multicolumn{4}{|c|}{ TABLA DE VOLUMEN (AGUAS ARRIBA) } \\
\hline ABSCISA & $\begin{array}{l}\text { Área de corte } \\
\qquad\left(\mathrm{m}^{2}\right)\end{array}$ & $\begin{array}{c}\text { Volumen de } \\
\text { corte }\left(\mathrm{m}^{3}\right)\end{array}$ & $\begin{array}{c}\text { Volumen de } \\
\text { corte acumulado } \\
\left(\mathrm{m}^{3}\right)\end{array}$ \\
\hline $0+000.00$ & 184.41 & 0.00 & 0.00 \\
\hline $0+025.00$ & 255.40 & $\mathbf{5 4 9 7 . 5 7}$ & $\mathbf{5 4 9 7 . 5 7}$ \\
\hline $0+050.00$ & 284.40 & 6747.47 & 12245.04 \\
\hline $0+075.00$ & 294.19 & 7232.40 & 19477.44 \\
\hline $0+100.00$ & 287.63 & 7272.77 & 26750.21 \\
\hline $0+125.00$ & 284.91 & 7429.06 & 34179.27 \\
\hline $0+150.00$ & 271.65 & 6957.03 & 41136.30 \\
\hline $0+175.00$ & 267.65 & 6741.30 & 47877.60 \\
\hline $0+200.00$ & 260.39 & 6600.54 & 54478.14 \\
\hline $0+225.00$ & 235.70 & 6201.19 & 60679.33 \\
\hline $0+250.00$ & 243.06 & 5984.48 & 66663.82 \\
\hline $0+275.00$ & 233.52 & 5957.20 & 72621.01 \\
\hline $0+300.00$ & 214.52 & 5600.50 & 78221.52 \\
\hline $0+325.00$ & 188.69 & 5040.11 & 83261.63 \\
\hline $0+350.00$ & 190.17 & 4727.70 & 87989.33 \\
\hline $0+375.00$ & 212.72 & 5036.13 & 93025.46 \\
\hline $0+400.00$ & 211.27 & 5299.92 & 98325.38 \\
\hline $0+425.00$ & 215.38 & 5333.14 & 103658.51 \\
\hline $0+44.84$ & 217.60 & 4294.23 & 107952.75 \\
\hline
\end{tabular}

Grafico 6: Tabla de volúmenes zona A 


\begin{tabular}{cccc}
\hline \multicolumn{4}{c}{ TABLA DE VOLUMEN (AGUAS ABAJO) } \\
\hline ABSCISA & $\begin{array}{c}\text { Área de corte } \\
\left(\mathrm{m}^{2}\right)\end{array}$ & $\begin{array}{c}\text { Volumen de } \\
\text { corte }\left(\mathrm{m}^{3}\right)\end{array}$ & $\begin{array}{c}\text { Volumen de } \\
\text { corte acumulado } \\
\left(\mathrm{m}^{3}\right)\end{array}$ \\
\hline $0+000.00$ & 0.00 & 0.00 & 0.00 \\
\hline $0+025.00$ & 224.00 & 2800.03 & 2800.03 \\
\hline $0+050.00$ & 215.68 & 5496.07 & 8296.10 \\
\hline $0+075.00$ & 199.05 & 5184.11 & 13480.21 \\
\hline $0+100.00$ & 202.97 & 5025.21 & 18505.42 \\
\hline $0+125.00$ & 205.89 & 5110.79 & 23616.21 \\
\hline $0+150.00$ & 214.85 & 5259.22 & 28875.43 \\
\hline $0+175.00$ & 229.07 & 5430.34 & 34305.77 \\
\hline $0+200.00$ & 226.20 & 5690.89 & 39996.66 \\
\hline $0+225.00$ & 210.49 & 5458.61 & 45455.27 \\
\hline $0+250.00$ & 200.30 & 5134.87 & 50590.14 \\
\hline $0+275.00$ & 204.55 & 5060.66 & 55650.80 \\
\hline $0+299.00$ & 193.97 & 4798.39 & 60449.19 \\
\hline
\end{tabular}

Grafico 7: Tabla de volúmenes zona B

\section{Resultado del ensayo de desgaste.}

Como un aporte a la investigación, se propuso realizar un ensayo de desgaste del material que se encuentra en el río Upano, este ensayo nos permitirá conocer el grado de abrasividad y desgaste que tienen los materiales al ser sometidos a abrasión, impacto y cizalla. Con los resultados obtenidos se determinará si cumple con las especificaciones ASTTHO T-96, que nos dice: si el material presenta un desgate menor a 0 ó igual al $50 \%$ presta las condiciones necesarias para ser usado como material de base para la construcción.

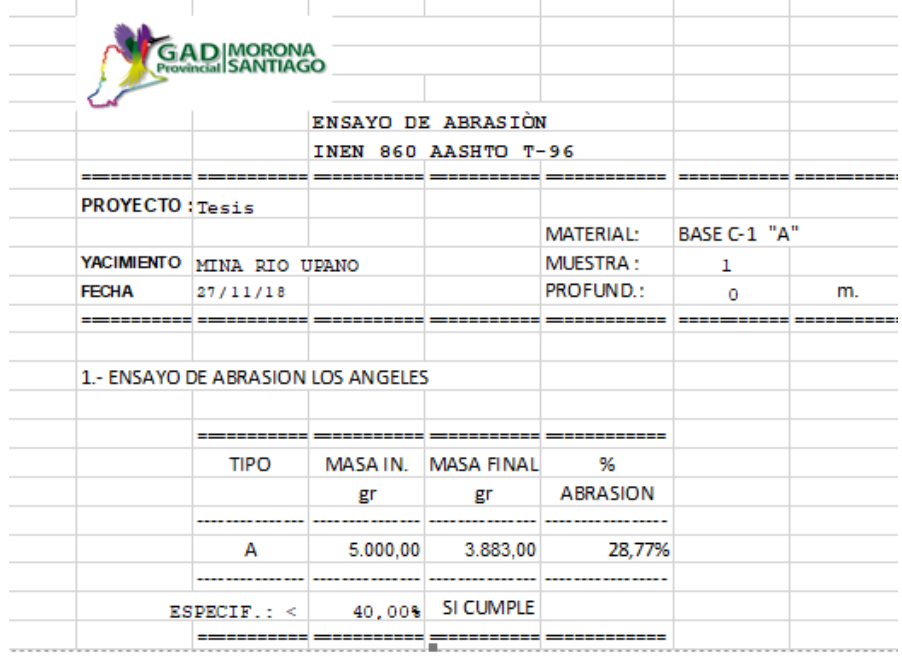




\section{Ciencia

Tabla 5: Resultado del ensayo de desgaste.

En la tabla se puede observar que en un total de 5000 gr de muestra fueron sometidas al ensayo de abrasividad por un período de tiempo de 15 minutos a una velocidad operacional de 30 a 33 RPM, el resultado de la muestra es de 3883 gr. El porcentaje de abrasión de la muestra es la resta entre la muestra inicial con la muestra final en porcentaje, dando como resultado un total del $28.77 \%$ de desgaste. De esta forma podemos comprobar que el material presenta las características normadas para ser usado como material de base.

\section{Resultado del ensayo granulométrico.}

Una vez realizado el ensayo granulométrico en un metro cúbico de material en dos puntos establecidos, se puedo observar la clasificación granulométrica presente en la muestra, en donde se clasificaron a los materiales en arenas finas, gruesas, así como cantos rodados o gravas y por último bloques a los pedazos no condicionadas de material.

\begin{tabular}{|c|c|c|c|c|c|c|}
\hline \multicolumn{7}{|c|}{ ORANUL OM ETRLA (A 8T M D422) } \\
\hline \multirow{2}{*}{ TAMIZ } & ABERTURA & PEBO RET. & PEBO RET_. & $\%$ & $\%$ & $\%$ \\
\hline & $(\mathrm{mm})$ & PARCIAL & ACUMULADO & RET ENIDO & QUE PA BA & ESTCLACADO \\
\hline & & & & & & \\
\hline & & & & & & \\
\hline & & & & & & \\
\hline & & & & & & \\
\hline & & & & & & \\
\hline & & & & & & \\
\hline & & & & & & \\
\hline N.2/8 & 9,5 & 0,48 & 0,5 & 19,12 & & \\
\hline $\mathrm{N} 040$ & $\overline{0,42}$ & 0,56 & $\overline{1,1}$ & 44,73 & 65,27 & \\
\hline $\mathrm{N}^{*} 60$ & 0.3 & 0,51 & 1,7 & 70,45 & 29,64 & \\
\hline $\mathrm{N} \cdot 90$ & 0,18 & - & 0,0 & 0,00 & 0,00 & \\
\hline $\mathrm{N}=100$ & 0,15 & 0,57 & 0,6 & 23,88 & 78,12 & \\
\hline$N^{2} 200$ & 0,07 & 0,15 & 0,7 & 30,17 & 69,88 & \\
\hline$\therefore \mathrm{Nand}$ & & & 2,2 & 93,50 & & \\
\hline TOTAL & & 2,37 & 2,4 & & & \\
\hline \multirow[t]{5}{*}{ PESO AN } & S DEL LAVA & $0=$ & \multirow{5}{*}{\multicolumn{2}{|c|}{ - gr }} & ÁRIDO & $\%$ \\
\hline & & & & & QRUEBA & 18,12 \\
\hline & & & & & MEDIA & 11,06 \\
\hline & & & & & FINO 8 & 69,32 \\
\hline & & & & & TOTAL \% & 100,00 \\
\hline
\end{tabular}

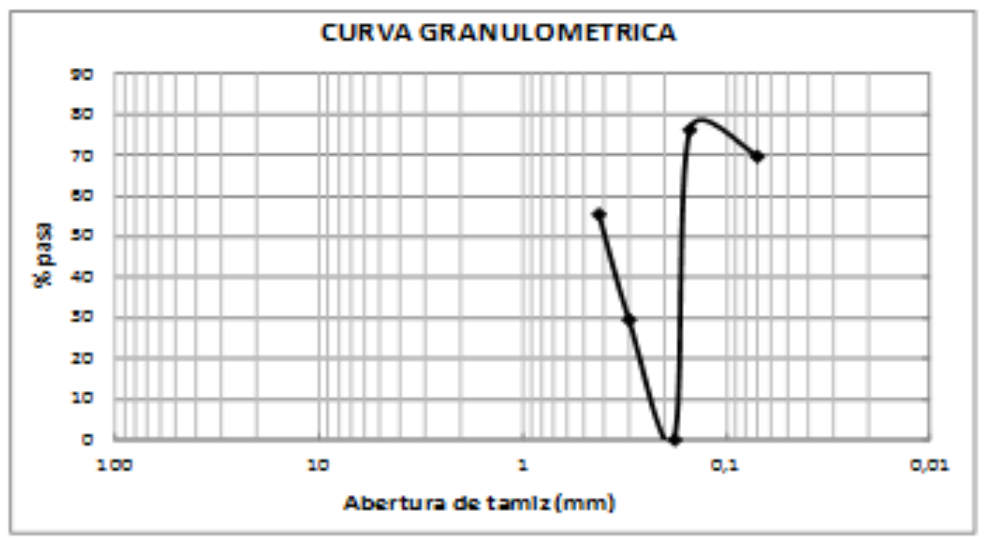

Tabla 6: Resultado del ensayo granulométrico

El material predomínate en este caso son los bloques de dimensiones mayores 10 hasta $60 \mathrm{~cm}$ y en un porcentaje reducido las arenas de tamaño medio y finas. Este ensayo es un 


\section{Ciencia

indicador teórico y práctico del tipo de material presente en el río, pero no resulta de mucha importancia debido a que los mineros locales utilizan plantas de trituración para la obtención del material de acuerdo a los requerimientos del cliente, utilizando primero una clasificación mediante cribas donde la abertura por metro lineal depende de la capacidad del setting de la chancadora, que van desde los $15 \mathrm{~cm}$ hasta los 25 de esta forma realizan la primera separación, luego trasladan el material hasta la trituradora primaria donde obtiene un producto final de triturado clase uno de un diámetro de hasta $4 \mathrm{~cm} \mathrm{y}$ además de arenas finas y gruesas

Una vez realizado el ensayo de granulométrica del $30 \%$ de la muestra que corresponde a un material menor a los $15 \mathrm{~cm}$; se puedo determinar que tenemos un $19.12 \%$ de arena gruesa, un $11.05 \%$ arena media y un $69.83 \%$ de arena fina

\section{Plan de manejo de explotación.}

De acuerdo a lo mencionado con anterioridad y a toda la información recopilada, se puede proponer un plan de manejo de explotación acorde a los parámetros establecidos por el Instructivo Municipal de Macas y al instructivo de maquinaria y equipos con capacidades de carga y producción en minería artesanal en el capítulo 3 Art. 5, donde establece la maquinaria a utilizar y la profundidad máxima permitida a excavar con estos límites y especificaciones de explotación y cuáles son los lineamientos a seguir para realizar dicha actividad, se establece una metodología de explotación.

En función de todas las especificaciones antes mencionadas, la forma más adecuada de explotar el material áridos y pétreos por los mineros artesanos en este tipo de ríos, es mediante franjas ubicadas a 10 metros y de forma perpendiculares al avance natural del río, con secciones no mayores a 3 metros de ancho por 5 de largo y con una profundidad máxima de 5.40 metros, debido a que el material que se extrae es de dimisiones variadas hasta un diámetro máximo de $30 \mathrm{~cm}$, los materiales superiores a esta dimensión, deberá ser utilizado como escolleras para proteger el cauce, evitando que este se desvié y provoque afecciones a las áreas donde se está extrayendo el material.

En función a los requerimientos y la demanda del material, el área minera deberá contar con un patio de stock y una zona de clasificación de material para separar las arenas del material grueso, de esta manera se obtiene una extracción muy ordenada, amigable con el medio ambiente y más importante aún, el material se repondrá de forma natural a medida que avanza la explotación.

Como un aporte adicional y de mucha importancia se cree necesario la construcción de ataguías al margen derecho del río, en zonas de mayor probabilidad de que el río desborde su cauce en crecentadas, debido al mal manejo de las zonas de explotación de material. Una de las características y ventajas que presentan este tipo de estructuras son que se pueden elaborar con el material sobre dimensionado que se extraer del mismo río en 


\section{Ciencia

forma de pilas de mayor a menor diámetro, de esta forma se crea una estructura natural que evitara que el río pierda su cauce a la hora de realizar la explotación, conservado de esta forma la topografía natural del mismo.

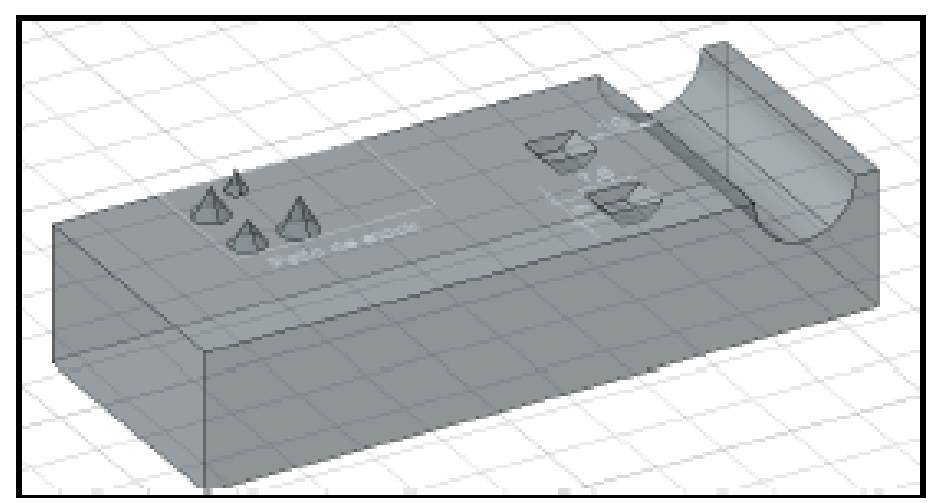

Grafico 8: Diseño explotación vista 3D

\section{CONCLUSIONES.}

- Mediante el levantamiento batimétrico del tramo seleccionado, se determinó que hasta una profundidad de 3.40 metros es viable realizar la extracción de material en el río, en las orillas la profundidad puede aumentar hasta un máximo de 4 metros.

- El porcentaje de desgaste según el ensayo de abrasividad es de $28.77 \%$ cumpliendo así con los parámetros establecidos por las normativas NEVI y ASTTHO T-96, considerándolo así material de buena calidad.

- En cuanto a los ensayos de granulometría, en un metro cúbico de material analizado tenemos: un $20.95 \%$ de arena, $9.05 \%$ de gravas y un $70 \%$ de bloques.

- La reposición de material se lo realiza de forma natural con las eventuales crecentadas que presenta el río a lo largo del año. De acuerdo al plan de desarrollo y ordenamiento territorial, 2012-2021 de la parroquia Macas, los meses de mayor precipitación son abril, julio y septiembre, donde el río Upano aumenta su caudal, por lo que ampliará su grado de reposición de material.

- Se determinó que el río en un período estimado de 9 meses, tiene una capacidad de reposición de $128982.13 \mathrm{~m} 3$ de material pétreo en un tramo de 60 metros de largo por 25 metros de ancho, dando así una capacidad de reposición mensual de $14331.13 \mathrm{~m} 3$, semestral de $85986.78 \mathrm{~m} 3$ y anual de $171973.50 \mathrm{~m} 3$; permitiendo que la explotación de material sea viable y no altere al río como sus reservas de material.

- El método de explotación acorde al sector es, mediante la extracción con franjas perpendiculares al cauce del río, de una extensión máxima de 3.40 metros de profundidad por 5 de largo. 


\section{Oiencia

\section{Referencias Bibliográficas.}

Baldock, J. (1982). Geología del Ecuador, División de investigación geológica minera. Quito - Ecuador.

Baby, P., Rivadeneira, M. \& Barragán, R. (2004). La cuenca oriente geológica y petróleo. Quito - Ecuador.

Smith, S. \& Sandwell, J. (1997). Metodología de la batimetría y su aplicación. MadridEspaña.

Calderón, M. (2002). Metodología de posicionamiento batimétrico. Barcelona - España. Corporación Universitaria de Dios. (2015). Ensayos de abrasividad. Bogotá - Colombia. Herrera, J. \& Pla, F. (2006). Características y métodos de explotación. Madrid - España. Herrera, J. \& Pla, F. (2009). Materiales de construcción. Madrid - España.

Litherland, M. A. (1994). The metamorphic belts of Quito. Quito - Ecuador.

Duarte, M. (2014). Materiales de construcción. Quito - Ecuador.

Gobierno Autónomo Descentralizado Del Cantón Morona. (2012). Clima y temperatura. Macas- Ecuador.

Universidad Sucre. (2007). Características de los ensayos granulométricos. MedellínColombia. 


\section{PARA CITAR EL ARTÍCULO INDEXADO.}

Mejia, M. A., \& Quintuña, P. (2019). Determinación de capacidad de reposición natural de material pétreo en el tramo del rio Upano denominado la barranca para un manejo de explotación. Ciencia Digital, 3(3.1), 409427. https://doi.org/10.33262/cienciadigital.v3i3.1.711

\section{Ciencia \\ Digital \\ Editorial}

El artículo que se publica es de exclusiva responsabilidad de los autores y no necesariamente reflejan el pensamiento de la Revista Ciencia Digital.

El artículo queda en propiedad de la revista y, por tanto, su publicación parcial y/o total en otro medio tiene que ser autorizado por el director de la Revista Ciencia Digital.
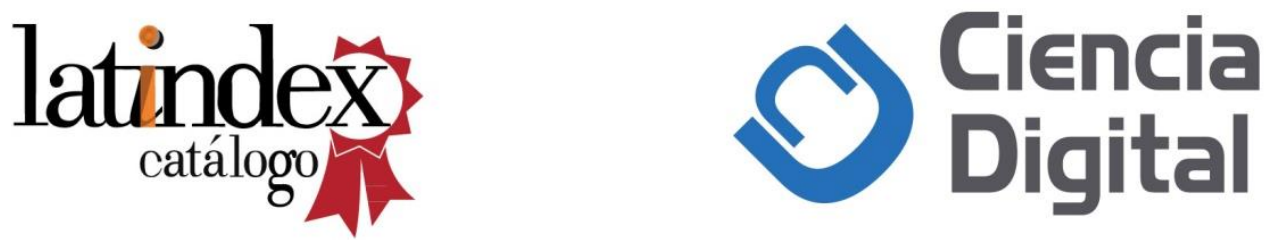